\title{
Oscillation of Second Order Nonlinear Differential Equations with a Damping Term
}

\author{
Xue $\mathrm{Mi}^{1,}$, , Ying Huang ${ }^{1,2}$, Desheng $\mathrm{Li}^{1}$ \\ ${ }^{1}$ School of Mathematics and System Sciences, Shenyang Normal University, Shenyang, Liaoning, P. R. China \\ ${ }^{2}$ School of Mathematics, Jilin University, Changchun, Jilin, P. R. China

\section{Email address:} \\ 13478211559@163.com (Xue Mi), huangyingmath@126.com (Ying Huang),dsl_6638@163.com (Desheng Li) \\ ${ }^{*}$ Corresponding author
}

\section{To cite this article:}

Xue Mi, Ying Huang, Desheng Li. Oscillation of Second Order Nonlinear Differential Equations with a Damping Term. Applied and Computational Mathematics. Vol. 5, No. 2, 2016, pp. 46-50. doi: 10.11648/j.acm.20160502.12

Received: February 5, 2016; Accepted: March 7, 2016; Published: March 25, 2016

\begin{abstract}
A class of second-order nonlinear differential equations with a damping term is investigated in this paper. By using the Riccati transformation technique and general weight functions, we obtain some new sufficient conditions for the oscillation of the equation. Our results improve and extend some known results. Two examples are given to illustrate the main results.
\end{abstract}

Keywords: Oscillation, Second Order Nonlinear Differential Equation, Damping Term, Riccati Transformation Technique, Weight Function

\section{Introduction}

In this paper we are concerned with the problem of oscillation of the nonlinear second order differential equation with a damping term

$$
\begin{gathered}
\left(r(t)\left(x^{\prime}(t)\right)^{\alpha}\right)^{\prime}+p(t)\left(x^{\prime}(t)\right)^{\alpha}+q(t) f(x(t))=0 \\
t \geq t_{0}>0
\end{gathered}
$$

Several assumptions are as follow:

(I) $r(t) \in C^{1}\left(\left[t_{0}, \infty\right),(0, \infty)\right), p(t), q(t) \in C\left(\left[t_{0}, \infty\right),(0, \infty)\right)$;

(II) $f(x) \in C(R, R)$, and $f(x) / x^{\beta} \geq k$, for some $k>0$ and for all $x(t) \neq 0 . \alpha \geq 1, \beta \geq 1$, and they are both quotients of odd positive integers.

Let $D=\left\{(t, s): t_{0} \leq s \leq t<+\infty\right\}, D_{0}=\left\{(t, s): t_{0} \leq s<t<+\infty\right\}$. We say the function $H \in C(D,[0,+\infty))$ belongs to a class $W_{\alpha}$ if:

(i) $H(t, t)=0$ for all $t \geq t_{0}, H(t, s)>0$ in $D_{0}$;

(ii) $H$ has a continuous and non-positive partial derivative in $D_{0}$ with respect to the second variable satisfying the condition

$$
\frac{\partial}{\partial s} H(t, s)=-h(t, s)(H(t, s))^{\alpha / \alpha+1},
$$

for some function $h \in L_{l o c}(D, R)$.

We shall consider the solutions of Equation (1) which are defined for all large $t$. A solution of Equation (1) is said to be oscillatory if it has arbitrarily large zeros, otherwise it is said to be non-oscillatory. Equation (1) is called oscillatory if all its solutions are oscillatory.

Recently, there are many authors who have investigated the oscillation for second order differential equations with a damping term, see [3-16] and the references are cited therein.

Wong [10] has studied the equation

$$
x^{\prime \prime}+p(t) x^{\prime}+q(t) f(x)=0 .
$$

Rogovchenko and Tuncay [7], M. Kirane and Yu. V. Rogovchenko [8], Yan [12] have obtained oscillation criteria of the following equation:

$$
\left(r(t) x^{\prime}(t)\right)^{\prime}+p(t) x^{\prime}(t)+q(t) f(x(t))=0 .
$$

Theorem A [8]. Assume that the function $f$ satisfies $\frac{f(x)}{x} \geq K>0$ for some constant $K$ and for all $x \neq 0$. Suppose 
further that the functions $h, H \in C(D,(-\infty,+\infty))$ are such that $H$ belongs to the class $\mathrm{P}$ and

$$
\frac{\partial}{\partial s} H(t, s)=-h(t, s)(H(t, s))^{1 / 2} \text {, for all }(t, s) \in D_{0} .
$$

Assume that there exists a function $g \in C^{1}\left(\left[t_{0}, \infty\right) ;(0, \infty)\right)$ such that

$$
\limsup _{t \rightarrow \infty} \frac{1}{H\left(t, t_{0}\right)} \int_{t_{0}}^{t}\left[H(t, s) \psi(s)-\frac{a(s) r(s)}{4} Q^{2}(t, s)\right] d s=\infty,
$$

where $a(s)=\exp \left(-\int^{s} g(u) d u\right)$

$$
\psi(s)=a(s)\left(K q(s)-p(s) g(s)-(r(s) g(s))^{\prime}+r(s) g^{2}(s)\right)
$$

and $Q(t, s)=h(t, s)+p(s)(r(s))^{-1}(H(t, s))^{1 / 2}$,

Then Eq.(3) is oscillatory.

More recently, Li et al [9] investigated oscillation criteria for the following equation:

$$
\left(r(t)\left(x^{\prime}(t)\right)^{\gamma}\right)^{\prime}+p(t)\left(x^{\prime}(t)\right)^{\gamma}+q(t) f(x(t))=0,
$$

where $\gamma \geq 1$ is a quotient of odd positive integers and $f(x) / x^{\gamma} \geq \mu$ for some $\mu>0$.

Theorem B [9]. Suppose that there exists a function $\rho \in C^{1}\left(\left(t_{0},+\infty\right), R\right)$ such that, for some $\beta \geq 1$ and for some $H \in W_{\gamma}$,

$\limsup _{t \rightarrow \infty} \frac{1}{H\left(t, t_{0}\right)} \int_{t_{0}}^{t}\left[H(t, s) \psi(s)-\frac{\beta^{\gamma}}{(\gamma+1)^{\gamma+1}} v(s) r(s) h^{\gamma+1}(t, s)\right] d s=\infty$

where

$$
\begin{gathered}
\psi(s)=v(s)\left(\mu q(s)-p(s) \rho(s)-(r(s) \rho(s))^{\prime}+r(s) \rho^{\gamma+1 / \gamma}(s)\right), \\
v(s)=\exp \left(-(\gamma+1) \int_{t_{0}}^{t}\left[\rho^{1 / \gamma}(s)-\frac{p(s)}{(\gamma+1) r(s)}\right] d s\right)
\end{gathered}
$$

and $Q(t, s)=h(t, s)+p(s)(r(s))^{-1}(H(t, s))^{1 / 2}$.

Then Eq. (4) is oscillatory.

Theorem C [9]. Suppose that there exists a function $H \in W_{\gamma}$,

$\rho \in C^{1}\left(\left(t_{0},+\infty\right), R\right)$ and $\phi \in C\left(\left(t_{0},+\infty\right), R\right)$ such that, for some $\beta \geq 1$ and for all $T \geq t_{0}$,

$$
0<\inf _{s \geq t_{0}}\left[\liminf _{t \rightarrow \infty} \frac{H(t, s)}{H\left(t, t_{0}\right)}\right] \leq+\infty
$$

and

$\limsup _{t \rightarrow \infty} \frac{1}{H\left(t, t_{0}\right)} \int_{t_{0}}^{t}\left[H(t, s) \psi(s)-\frac{\beta^{\gamma}}{(\gamma+1)^{\gamma+1}} v(s) r(s) h^{\gamma+1}(t, s)\right] d s \geq \phi(T)$ where $\psi(s)$ and $v(s)$ are as in theorem B. If

$$
\int_{t_{0}}^{+\infty}\left(\frac{\phi_{+}(s)}{v(s) r(s)}\right)^{1 / \gamma} d s=+\infty
$$

where $\phi_{+}(t)=\max \{\phi(t), 0\}$, then Eq.(4) is oscillatory.

It is obvious that (2), (3) and (4) are special cases of Eq. (1).

Motivated by the idea of $\mathrm{Li}$ [9], in this paper we obtain, by using a generalized Riccati technique due to Li [9], several new interval criteria for oscillation, that is, criteria given by the behavior of equation (1) on $\left[t_{0}, \infty\right)$. Our results improve and extend the results of $\mathrm{Li}$ [9], Rogovchenko [3, 7, 8], and Grace [16]. Finally, several examples are inserted to illustrate the main results.

\section{Lemmas}

Lemma 1 . Let $\lambda \geq 1$ be a ratio of two odd numbers. Then,

$$
A^{1+1 / \lambda}-(A-B)^{1+1 / \lambda} \leq \frac{B^{1 / \lambda}}{\lambda}[(\lambda+1) A-B]
$$

Lemma 2. Let $C \neq 0, D>0, u>0$ and $\lambda>0$, then

$$
D u-C u^{\frac{\lambda+1}{\lambda}} \leq\left(\frac{\lambda^{\lambda}}{(\lambda+1)^{\lambda+1}}\right) \frac{D^{\lambda+1}}{C^{\lambda}} .
$$

\section{Conclusions}

Theorem 1. Suppose that there exists a function $g(t) \in C^{1}\left(\left[t_{0}, \infty\right), R\right)$ such that, for some $m \in[0,1]$ and for some $H \in W_{\alpha}$,

$$
\limsup _{t \rightarrow \infty} \frac{1}{H\left(t, t_{0}\right)} \int_{t_{0}}^{t}\left[H(t, s) \psi(s)-\frac{h^{\alpha+1}(t, s) r(s) v(s)}{(\alpha+1)^{\alpha+1}}\right] d s=\infty
$$

where $\quad v(t)=\exp \left\{\int_{t_{0}}^{t}\left[\frac{p(s)}{r(s)}-(\alpha+1) g^{\frac{1}{\alpha}}(s)\right] d s\right\} \quad$ and $\psi(t)=m^{\beta-\alpha} k v(t) q(t)+g^{\frac{\alpha+1}{\alpha}}(t) v(t) r(t)-g(t) p(t) r(t)$

$-v(t)(r(t) g(t))^{\prime}$,

Then, equation (1) is oscillatory.

Proof. To obtain a contradiction, suppose that $x(t)$ is a non-oscillatory solution of Eq. (1) and let $t_{1} \geq t_{0}$ such that $x(t) \neq 0$ for all $t \geq t_{1}$. Without loss of generality, we may assume that $x(t)>0$ for all $t \geq t_{1}$ since the similar argument holds also for $x(t)$ eventually negative. We define a generalized Riccati substitution by

$$
u(t)=v(t) r(t)\left[\left(\frac{x^{\prime}(t)}{x(t)}\right)^{\alpha}+g(t)\right], t \geq t_{1}
$$


Differentiating (8) and using (1), we obtain

$$
\begin{gathered}
u^{\prime}(t)=\frac{v^{\prime}(t)}{v(t)} u(t)+v(t)(r(t) g(t))^{\prime}-\frac{p(t) u(t)}{r(t)}+p(t) g(t) v(t) \\
-v(t) q(t) \frac{f(x(t))}{(x(t))^{\alpha}}-\alpha v(t) r(t)\left(\frac{x^{\prime}(t)}{x(t)}\right)^{\alpha+1}-\alpha v(t) r(t)\left(\frac{x^{\prime}(t)}{x(t)}\right)^{\alpha+1} \\
\leq \frac{v^{\prime}(t)}{v(t)} u(t)+v(t)(r(t) g(t))^{\prime}-\frac{p(t) u(t)}{r(t)}+p(t) g(t) v(t) \\
-k v(t) q(t)(x(t))^{\beta-\alpha}-\alpha v(t) r(t)\left(\frac{u(t)}{v(t) r(t)}-g(t)\right)^{\frac{\alpha+1}{\alpha}}, \quad(9)
\end{gathered}
$$

where $x(t)$ is a continuous function and $x(t)>0$, so there exist $t_{2} \geq t_{1}$ and $m \in[0,1]$ such that $x(t) \geq m$, for all $t \geq t_{2}$. By lemma 1, let $A=\frac{u(t)}{v(t) r(t)}, B=g(t)$, then

$$
\begin{gathered}
{\left[\frac{u(t)}{v(t) r(t)}-g(t)\right]^{\frac{\alpha+1}{\alpha}} \geq\left(\frac{u(t)}{v(t) r(t)}\right)^{\frac{\alpha+1}{\alpha}}} \\
-\frac{g^{\frac{1}{\alpha}}(t)}{\alpha}\left[(\alpha+1) \frac{u(t)}{v(t) r(t)}-g(t)\right]
\end{gathered}
$$

Thus, (9) and (10) yield

$$
u^{\prime}(t) \leq-\psi(t)-\alpha\left(\frac{u^{\alpha+1}(t)}{v(t) r(t)}\right)^{\frac{1}{\alpha}}
$$

Multiplying the both sides of (11) by $H(t, s)$ and integrating the inequality from $t_{2}$ to $t$, we obtain, for all $t \geq t_{2}$

$$
\begin{gathered}
\int_{t_{2}}^{t} H(t, s) \psi(s) d s+\alpha \int_{t_{2}}^{t} \frac{H(t, s)}{(r(s) v(s))^{\frac{1}{\alpha}}} u^{\frac{\alpha+1}{\alpha}}(s) d s \\
-\int_{t_{2}}^{t} h(t, s) H^{\frac{\alpha}{\alpha+1}}(t, s) u(s) d s
\end{gathered}
$$

$\leq H\left(t, t_{2}\right) u\left(t_{2}\right)-2 \int_{t_{2}}^{t} h(t, s) H^{\frac{\alpha}{\alpha+1}}(t, s) u(s) d s$.

Let $C=\alpha \frac{H(t, s)}{(r(s) v(s))^{\frac{1}{\alpha}}}, \quad D=h(t, s) H^{\frac{\alpha}{\alpha+1}}(t, s), \quad u=u(t)$ and $\lambda=\alpha$, by lemma 2 , we have

$$
\begin{gathered}
\int_{t_{2}}^{t} H(t, s) \psi(s) d s-\int_{t_{2}}^{t} \frac{h^{\alpha+1}(t, s) v(s) r(s)}{(\alpha+1)^{\alpha+1}} d s \leq H\left(t, t_{2}\right)\left|u\left(t_{2}\right)\right| \\
\leq H\left(t, t_{0}\right)\left|u\left(t_{2}\right)\right| .
\end{gathered}
$$

Thus,

$$
\begin{gathered}
\int_{t_{0}}^{t}\left[H(t, s) \psi(s)-\frac{h^{\alpha+1}(t, s) v(s) r(s)}{(\alpha+1)^{\alpha+1}}\right] d s \\
\quad \leq H\left(t, t_{0}\right)\left[\left|u\left(t_{2}\right)\right|+\int_{t_{0}}^{t}|\psi(s)| d s\right] .
\end{gathered}
$$

Hence,

$$
\begin{gathered}
\limsup _{t \rightarrow \infty} \frac{1}{H\left(t, t_{0}\right)} \int_{t_{0}}^{t}\left[H(t, s) \psi(s)-\frac{h^{\alpha+1}(t, s) r(s) v(s)}{(\alpha+1)^{\alpha+1}}\right] d s \\
\leq\left|u\left(t_{2}\right)\right|+\int_{t_{0}}^{t}|\psi(s)| d s<\infty,
\end{gathered}
$$

which contradicts (7). The proof is complete.

Theorem 2. Suppose that there exist functions $H \in W_{\alpha}$, $g(t) \in C^{1}\left(\left[t_{0}, \infty\right), R\right)$, and $\phi(t) \in C\left(\left[t_{0}, \infty\right), R\right)$ such that, for all $T \geq t_{0}$,

$$
0<\inf _{s \geq t_{0}}\left[\liminf _{t \rightarrow \infty} \frac{H(t, s)}{H\left(t, t_{0}\right)}\right] \leq+\infty
$$

and

$$
\limsup _{t \rightarrow \infty} \frac{1}{H(t, T)} \int_{T}^{t}\left[H(t, s) \psi(s)-\frac{h^{\alpha+1}(t, s) r(s) v(s)}{(\alpha+1)^{\alpha+1}}\right] d s \geq \phi(T)
$$

where $\psi$ and $v$ are as in Theorem 1 . If

$$
\int_{t_{0}}^{\infty} \frac{\phi_{+}(s) h(t, s)}{(H(t, s))^{\frac{1}{\alpha+1}}} d s=\infty
$$

where $\phi_{+}(t)=\max \{\phi(t), 0\}$, then equation (1) is oscillatory.

Proof. As in Theorem 1, without loss of generality we may assume that there exists a solution $x(t)$ of Eq. (1) such that $x(t)>0$ on $\left[t_{1}, \infty\right)$ for some $t_{1} \geq t_{0}$. Defining again the function $u(t)$ by (8), we arrive at (12) which implies, for all $t>t_{2}$

$$
\begin{aligned}
& \int_{t_{2}}^{t} H(t, s) \psi(s) d s-\int_{t_{2}}^{t} \frac{h^{\alpha+1}(t, s) v(s) r(s)}{(\alpha+1)^{\alpha+1}} d s \\
& \leq H\left(t, t_{2}\right) u\left(t_{2}\right)-2 \int_{t_{2}}^{t} h(t, s) H^{\frac{\alpha}{\alpha+1}}(t, s) u(s) d s .
\end{aligned}
$$

By (14), we have

$$
\limsup _{t \rightarrow \infty} \frac{1}{H\left(t, t_{2}\right)} \int_{t_{2}}^{t}\left[H(t, s) \psi(s)-\frac{h^{\alpha+1}(t, s) r(s) v(s)}{(\alpha+1)^{\alpha+1}}\right] d s \geq \phi\left(t_{2}\right) .
$$

Thus for all $t>t_{2}$, 
$\phi\left(t_{2}\right) \leq u\left(t_{2}\right)-2 \liminf _{t \rightarrow \infty} \frac{1}{H\left(t, t_{2}\right)} \int_{t_{2}}^{t} h(t, s) H^{\frac{\alpha}{\alpha+1}}(t, s) u(s) d s$.

$$
\phi\left(t_{2}\right) \leq u\left(t_{2}\right)
$$

Consequently,

$$
2 \liminf _{t \rightarrow \infty} \frac{1}{H\left(t, t_{2}\right)} \int_{t_{2}}^{t} h(t, s) H^{\frac{\alpha}{\alpha+1}}(t, s) u(s) d s \leq u\left(t_{2}\right)-\phi\left(t_{2}\right)<+\infty
$$

Assume that

$$
\int_{t_{2}}^{+\infty} \frac{u(s) h(t, s)}{(H(t, s))^{\frac{1}{\alpha+1}}} d s=+\infty
$$

By (13), there exists a $\lambda>0$ such that

$$
\liminf _{t \rightarrow \infty} \frac{H(t, s)}{H\left(t, t_{0}\right)}>\lambda
$$

By (18), for any positive constant $\gamma$, there exists a $t_{3}>t_{2}$ such that for all $t>t_{3}$,

$$
\int_{t_{2}}^{+\infty} \frac{u(s) h(t, s)}{(H(t, s))^{\frac{1}{\alpha+1}}} d s \geq \frac{\gamma}{\lambda}
$$

Then

$$
\begin{gathered}
\frac{1}{H\left(t, t_{2}\right)} \int_{t_{2}}^{t} h(t, s) H^{\frac{\alpha}{\alpha+1}}(t, s) u(s) d s \\
=\frac{1}{H\left(t, t_{2}\right)} \int_{t_{2}}^{t} \frac{u(s) h(t, s)}{H^{\frac{1}{\alpha+1}}(t, s)} H(t, s) d s \\
=\frac{1}{H\left(t, t_{2}\right)} \int_{t_{2}}^{t} H(t, s) d\left[\int_{t_{2}}^{s} \frac{u(\xi) h(t, \xi)}{H^{\frac{1}{\alpha+1}}(t, \xi)} d \xi\right] \\
=\frac{1}{H\left(t, t_{2}\right)} \int_{t_{2}}^{t}\left[\int_{t_{2}}^{s} \frac{u(\xi) h(t, \xi)}{H^{\frac{1}{\alpha+1}}(t, \xi)} d \xi\right]\left(-\frac{\partial H(t, s)}{\partial s}\right) d s \\
\geq \frac{1}{H\left(t, t_{2}\right)} \frac{\gamma}{\lambda} \int_{t_{3}}^{t}\left(-\frac{\partial H(t, s)}{\partial s}\right) d s=\frac{\gamma}{\lambda} \frac{H\left(t, t_{3}\right)}{H\left(t, t_{2}\right)} \geq \frac{\gamma}{\lambda} \frac{H\left(t, t_{3}\right)}{H\left(t, t_{0}\right)} .
\end{gathered}
$$

By (19), there exists a $t_{4}>t_{3}$ such that, for all $t>t_{4}$,

$$
\frac{H\left(t, t_{3}\right)}{H\left(t, t_{0}\right)}>\lambda
$$

which implies that

$$
\frac{1}{H\left(t, t_{2}\right)} \int_{t_{2}}^{t} h(t, s) H^{\frac{\alpha}{\alpha+1}}(t, s) u(s) d s>\gamma, t>t_{4} .
$$

Since $\gamma$ is an arbitrary positive constant,

$$
2 \liminf _{t \rightarrow \infty} \frac{1}{H\left(t, t_{2}\right)} \int_{t_{2}}^{t} h(t, s) H^{\frac{\alpha}{\alpha+1}}(t, s) u(s) d s=+\infty
$$

and that contradicts (15).

Thus

$$
\int_{t_{2}}^{+\infty} \frac{u(s) h(t, s)}{(H(t, s))^{\frac{1}{\alpha+1}}} d s<+\infty
$$

and by (16),

$$
\int_{t_{0}}^{\infty} \frac{\phi_{+}(s) h(t, s)}{(H(t, s))^{\frac{1}{\alpha+1}}} d s \leq \int_{t_{0}}^{\infty} \frac{u(s) h(t, s)}{(H(t, s))^{\frac{1}{\alpha+1}}} d s<\infty,
$$

which contradicts (15). This complete the proof.

\section{Examples}

Example 1. Consider the following equation

$$
\begin{gathered}
\left(\frac{1}{t}\left(x^{\prime}(t)\right)^{\alpha}\right)^{\prime}+t\left(x^{\prime}(t)\right)^{\alpha}+\left(1-\frac{1}{t} g^{\frac{\alpha+1}{\alpha}}(t)+\frac{1}{t} g(t)\right. \\
\left.+\frac{1}{t} g^{\prime}(t)-\frac{1}{t^{2}} g(t)\right)(x(t))^{\beta}=0, t \geq 1
\end{gathered}
$$

where $\alpha \geq 1, \beta \geq 1$ are both quotients of odd positive integers, $k=1, m=1$ and $g(t)=\left(\frac{t^{2}-t^{-1}}{\alpha+1}\right)^{\alpha}$.

Let $H(t, s)=(t-s)^{2}$. Then $h(t, s)=2(t-s)^{(1-\alpha) /(1+\alpha)}$, $v(t)=t$ and $\psi(t)=t$. We have

$$
\begin{aligned}
& \limsup _{t \rightarrow \infty} \frac{1}{H\left(t, t_{0}\right)} \int_{t_{0}}^{t}\left[H(t, s) \psi(s)-\frac{h^{\alpha+1}(t, s) r(s) v(s)}{(\alpha+1)^{\alpha+1}}\right] d s \\
& =\limsup _{t \rightarrow \infty} \frac{1}{(t-1)^{2}} \int_{1}^{t}\left[(t-s)^{2} s-\frac{2^{\alpha+1}(t-s)^{1-\alpha}}{(\alpha+1)^{\alpha+1}}\right] d s=\infty
\end{aligned}
$$

By theorem 1, Eq. (21) is oscillatory.

Example 2. Consider the following equation

$$
\left(\sin t\left(x^{\prime}(t)\right)^{\alpha}\right)^{\prime}+\frac{\sin t}{t}\left(x^{\prime}(t)\right)^{\alpha}+(x(t))^{\beta}=0, t \geq 1
$$


where $\alpha \geq 1, \beta \geq 1$ are both quotients of odd positive integers, $k=1$ and $m=1$. Let $H(t, s)=(t-s)^{2}$ and $g(t)=0$. Then $h(t, s)=2(t-s)^{(1-\alpha) /(1+\alpha)}, \quad v(t)=t \quad$ and $\psi(t)=t$.

We have

$$
\begin{gathered}
\limsup _{t \rightarrow \infty} \frac{1}{H(t, T)} \int_{T}^{t}\left[H(t, s) \psi(s)-\frac{h^{\alpha+1}(t, s) r(s) v(s)}{(\alpha+1)^{\alpha+1}}\right] d s \\
=\limsup _{t \rightarrow \infty} \frac{1}{(t-T)^{2}} \int_{T}^{t}\left[(t-s)^{2} s-\frac{2^{\alpha+1}(t-s)^{(1-\alpha)} s \sin s}{(\alpha+1)^{\alpha+1}}\right] d s \\
\geq \limsup _{t \rightarrow \infty} \frac{1}{(t-T)^{2}} \int_{T}^{t}(t-s)^{2} s d s=\phi(T) .
\end{gathered}
$$

It is easy to verify that (15) is satisfied. Hence, Eq. (22) is oscillatory by theorem 2 .

\section{References}

[1] Y. V. Rogovchenko, "Note on 'Oscillation criteria for second order linear differential equations'," J. Math. Anal. Appl. 203 (1996) 560-563.

[2] Y. V. Rogovchenko, "Oscillation criteria for second order nonlinear perturbed differential equations," J. Math. Anal. Appl. 215 (1997) 334-357.

[3] Y. V. Rogovchenko, "Oscillation theorems for second order equations with damping," Nonlinear Anal. 41 (2000) 1005-1028.

[4] S. P. Rogovchenko, Y. V. Rogovchenko, "Oscillation of differential equations with damping," Dyn. Contin. Discrete Impuls. Syst. Ser. A Math. Anal. 10 (2003) 447-461.

[5] S. P. Rogovchenko, Y. V. Rogovchenko, "Oscillation results for general second-order differential equations with damping term," J. Math. Anal. Appl. 279 (2003) 139-152.

[6] Y. V. Rogovchenko, Tuncay F., "Oscillation criteria for second-order nonlinear differential equations with damping," Nonlinear Anal. 69 (2008) 208-221.

[7] Y. V. Rogovchenko, F. Tuncay, "Oscillation theorems for a class of second order nonlinear differential equations with damping," Taiwanese J. Math.13 (2009) 1909-1928.

[8] M. Kirane and Y. V. Rogovchenko, "On oscillation of nonlinear second order differential equation with damping term," Appl. Math. Comput., 117 (2001) 177-192.
[9] T. X. Li, Y. V. Rogovchenko, S. H. Tang., "Oscillation criteria for second-order nonlinear differential equations with damping," Math. Slovaca 64(2014) No. 5, 1227-1236.

[10] J. S. W. Wong, "On Kamenev-type oscillation for second order differential equations with damping," J. Math. Anal. Appl. 248 (2001) 244-257.

[11] J. Yan, "A note on an oscillation criterion for an equation with damped term," Proc. Amer. Math. Soc. 90 (1984) 277-280.

[12] J. Yan, "Oscillation theorems for second order linear differential equations with damping," Proc. Amer. Math. Soc. 98 (1986) 276-282.

[13] C. C. Yeh, "Oscillation theorems for nonlinear second order differential equations with damping term," Proc. Amer. Math. Soc. 84 (1982) 397-402.

[14] S. R. Grace, B. S. Lalli, "Oscillation theorems for second order superlinear differential equations with damping," J. Austral. Math. Soc. Ser. A 53(1992) 156-165.

[15] S. R. Grace, B. S. Lalli, C. C. Yeh, "Oscillation theorems for nonlinear second order differential equations with a nonlinear damping term,” SIAM J. Math. Anal. 15 (1984) 1082-1093.

[16] S. R. Grace, B. S. Lalli, C. C. Yeh, "Addendum: Oscillation theorems for nonlinear second order differential equations with a nonlinear damping term," SIAM J. Math. Anal. 19 (1988) 1252-1253.

[17] X. J. Wang, G. H. Song, "Oscillation Theorems for a class of nonlinear second order differential equations with damping," Advances in Pure Mathematics. 3(2013) 226-233.

[18] E. Tunc, H. Avci, "Oscillation criteria for a class of second order nonlinear differential equations with damping," Bulletin of Mathematical Analysis and Applications. 4 (2012) 40-50.

[19] E. Tunc, "A note on the oscillation of second order differential equations with damping," J. Comput. Anal. Appl. 12 (2010) 444-453.

[20] E. Tunc, H. Avci, "New oscillation theorems for a class of second order damped nonlinear differential equations," Ukrainian Math. J. 63 (2012) 1441-1457.

[21] A. A. Salhin, U. K. S. Din, R. R. Ahmad, M. S. M. Noorani, "Some oscillation criteria for a class of second order nonlinear damped differential equations," Applied Mathematics and Computation, 247 (2014) 962-968.

[22] A. A. Salhin, U. K. S. Din, R. R. Ahmad, M. S. M. Noorani, "Set of oscillation criteria for second order nonlinear forced differential equations with damping," Discrete Dynamics in Nature and Society, Volume 2014, Article ID 545189, 13 pages. 